\title{
Formación Integral en competencias para Impresión 3D
}

Rut Benavente ${ }^{a}$, Iván Patrao ${ }^{\mathrm{a}}$, Graham Small ${ }^{\mathrm{b}}$ y Nikos Tsianos ${ }^{\mathrm{c}}$

a rbenavente@florida-uni.es, ipatrao@florida-uni.es , Unidad de ingenería, Florida Centre de Formació, 46470, Catarroja, España.

b.small@amrc.co.uk, AMRC Training Centre, University of Sheffield, United Kingdom.

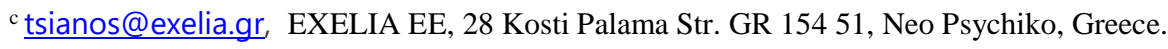

\begin{abstract}
Competences, skills and habilities adquired by students in the learning process must be determined by the learning outcomes, previously defined. In the 3D-PRISM European Project, the learning outcomes for 3D-printing students have been stablished.

The process for determining the learning outcomes did take in consideration the opinion of University and Professionals of the manufacturing industry. The proposed methodology comprises 4 phases: creation of the profiles for the professionals in the 3D-printing industry, creation of the questionnaire based in previous documental research, on-line questionnaire and data collection and processing
\end{abstract}

Keywords: Learning outcomes, skills, ETC's, ECVET's, $3 D$ printing

\section{Resumen}

Los conocimientos, competencias y/o habilidades adquiridas por el alumnado en el proceso de formación, han de estar determinadas por los resultados de aprendizaje previamente establecidos.

En el Proyecto Europeo 3D-PRISM se han establecido los resultados de aprendizaje en materia de Impresión 3D. El proceso ha sido llevado a cabo mediante el dialogo establecido entre la Universidad y la sociedad representada por expertos profesionales y docentes en la materia. La metodología propuesta en este trabajo se basa en cuatro fases: elaboración de los perfiles de los expertos en materia de impresión 3D, elaboración de un cuestionario basado en una investigación documental previa, distribución on-line de los cuestionarios y la recopilación de los datos obtenidos y tratamiento de los datos.

Palabras clave: Resultados de aprendizaje, Competencias, ECT's, ECVET's, Impresión 3D 


\section{Introducción}

\subsection{Conocimientos, Habilidades y Competencias}

En el desarrollo de un programa docente deben cubrirse tres pilares básicos: Conocimientos, Habilidades y Competencias (European Communities, 2008; Gonçalves, 2013).

- Conocimientos: Resultado de la información asimilada a través del aprendizaje Se consideran "conocimientos" los principios básicos, teoremas y desarrollos teóricoprácticos que forman parte de una determinada área de estudio.

- Habilidades: Capacidad adquirida para aplicar el concimiento y usar los recursos disponibles para llevar a término una tarea y resolver problemas.

- Competencias: Hace referencia a la capacidad para el uso del conocimiento, las habilidades y habilidades personales y sociales a la hora de resolver problemas personales y profesionales. Las competencias pueden entenderse como una forma de responsabilidad y autonomía.

El alumno habrá alcanzado el grado de aprendizaje óptimo cuando haya interiorizado conocimientos y habilidades que le permitan desarrollarse tanto en el campo de estudio como en otros relacionados, habiendo adquirido autonomía a la hora de afrontar problemas de tipo innovador, es decir, cuando haya desarrollado las competencias trabajadas en el curso.

La adquisición por parte del alumno de conocimientos, habilidades y competencias se comprueba durante el desarrollo del curso mediante los "Resultados de aprendizaje". Así, los "Resultados de aprendizaje" se utilizan para definir de forma precisa qué se espera enseñar en un curso. Se centran en la visión del alumno, especificando qué será capaz de hacer una vez finalizado (European Union, 2011). Un resultado de aprendizaje debe definir aquello que una persona sabrá, entenderá y será capaz de hacer al completar el proceso de aprendizaje.

Los Resultados de aprendizaje se enuncian basándose en las siguientes reglas:

- Se debe usar un verbo activo para expresar qué se espera que los estudiantes sean capaces de hacer.

- Se debe especificar a qué habilidad/es hace referencia cada resultado de aprendizaje

- Se debe especificar de qué modo se demostrará el cumplimiento de los resultados de aprendizaje.

En necesario definir cómo se medirá la carga de trabajo asociada a cada resultado de aprendizaje. A nivel europeo se han definido varios sistemas de créditos asociados a la carga de trabajo asociada a cada resultado de aprendizaje: ECTS y ECVET, descritos a continuación. 


\subsection{Sistema Europeo de Transferencia y Acumulación de Créditos (ECTS) y Sistema de Créditos en Educación y Formación Vocacional (ECVET)}

El Sistema Europeo de Transferencia y Acumulación de Créditos (European Credit Transfer and Accumulation System, ECTS) constituye en la actualidad una pieza clave del Espacio Europeo de Educación (European Higher Education Area, EHEA). En la actualidad el sistema basado en créditos ECTS es utilizado en todos los programas de estudios superiores del EHEA, aunque es aplicable a todo tipo de programas educativos, tanto presenciales como a distancia; pues traslada la visión del programa de estudios desde una visión centrada en el profesor a una visión centrada en el estudiante, siendo este uno de los pilares básicos de EHEA.

Los créditos ECTS expresan el volumen de carga de trabajo para cada resultado de aprendizaje. La carga de trabajo debe reflejar la totalidad de horas necesarias para alcanzar un resultado de aprendizaje: presenciales, trabajo en aula, en casa, prácticas, talleres, etc.

Por otro lado, el Sistema de Créditos en Educación y Formación Vocacional (Credit System in Vocational and Educational Training, ECVET) pretende servir de ayuda para la transferencia, reconocimiento y acumulación de resultados de aprendizaje, facilitando itinerarios formativos personalizados. Una mejor comprensión de los resultados de aprendizaje que acumula un individuo llevará, así, a una mayor movilidad internacional dentro de las fronteras de la Unión Europea, como se ha desmostrado en recientes ensayos de implantación del sistema ECVET (Cedefop 2013; European Commission 2014; Cedefop 2016).

Aunque la recomendación de la Unión Europea (European Parliament and Council of the European Union, 2009) es que se apliquen los ECVET a todas las titulaciones relacionadas con la formación vocacional y educacional (VET), la experiencia ha demostrado que, en las instituciones de educación superior, la aplicación práctica de este sistema encuentra una firme oposición.

Así, aunque el sistema ECVET todavía no ha sido implementado de forma masiva en todos los estados miembros de La Unión Europea, la aplicación de este sistema en educación superior parece ser, en el mejor de los casos, limitada. De hecho, los proveedores de formación VET de tercer nivel siguen prefiriendo un enfoque basado en ECTS, debido proincipalmente a su metodología más sencilla y a que, en educación superior, la ponderación de la carga de trabajo es un factor clave. Esto resuleve el problema de estructurar los curriculum docentes y agendas de profesores y alumnos, además de dar al estudiante una idea de la carga de trabajo que requiere cada curso ofertado.

Aunque podría parecer que un enfoque basado en ECVET es poco compatible con uno basado en ECTS, se ha demostrado que realizar una planificación basada en resultados de aprendizaje facilita enormemente la conversion bilateral entre ambos sistemas.

En este trabajo se presenta el proceso de identificación de los resultados de aprendizaje de un programa formativo en impresión 3D. Debido a la alta demanda de nuevos profesionales formados en esta técnica, se ha contado con la doble colaboración Universidad-Sociedad a la hora de determinar qué resultados de aprendizaje es necesario cubrir en esta formación. 


\section{Objetivos}

Mediante el díalogo establecido entre los expertos del sector de la fabricación aditiva (impresión 3D) y la Universidad, se pretende llegar a un entendimiento de lo que la sociedad demanda de los estudiantes egresados, de manera que se pueda establecer un perfil con las competencias perfectamente desarrolladas.

Por lo tanto, el principal objetivo de este trabajo se resume en:

- Establecer los resultados de aprendizaje en la formación sobre impresión 3D.

Para alcanzar el objetivo principal se han cubierto los siguientes objetivos secundarios:

- Apoyar la formación para el desarrollo de habilidades necesarias en el ámbito de la impresión 3D, mediante metodologías de formación específicas.

- Identificar nuevos perfiles en el sector de la fabricación aditiva, para facilitar y agilizar la integración de nuevas habilidades y cualificaciones en los alumnos en formación.

- Ayudar a aplicar las políticas de la UE para la cualificación, la transparencia, la movilidad y el renacer de la industria europea a través de puestos de trabajos seguros y sostenibles.

\section{Desarrollo de la innovación}

La metodología propuesta en este trabajo se llevó a cabo en cuatro fases. En la primera fase se elaboró el perfil de los expertos en materia de impresión 3D que formarán parte del estudio. En la segunda fase se llevó a cabo una investigación documental que culminó en la elaboración del cuestionario mediante el cual los expertos del sector, definirían las competencias y habilidades requeridas para el alumnado tanto en fase de preparación como en fase de producción. La tercera fase consistió en la distribución de los cuestionarios y la recopilación de los datos obtenidos. Por último, el tratamiento de los datos, originó los perfiles que demanda la sociedad para los expertos en material de fabricación 3D.

\subsection{Fase primera: Perfil del experto}

Las competencias adquiridas durante el periodo de formación del alumnado han de ser establecidas por la sociedad que les va a contratar. Es, por lo tanto, necesario consultar a la sociedad sobre qué competencias han de ser cubiertas. El papel de la sociedad, tendrá que ser cubierto por expertos en la materia.

Se consultó a expertos profesionales y expertos docentes. Como experto profesional, se engloban las personas que trabajan en empresa privada, tanto por cuenta propia como por cuenta ajena, en el ámbito de la fabricación aditiva. El experto docente, comprende a los profesionales docentes de la Universidad Pública, que imparten clases relacionadas con la fabricación asistida. 
En el perfil de expertos profesionales se ha logrado contactar con representantes de multinacionales, pymes y autónomos, así como fabricantes y vendedores de maquinaria y equipamiento, cubriendo todo el espectro de empresas dedicadas a la fabricación aditiva.

En el perfil de expertos docentes se ha logrado contactar tanto con titulares de Universidad como con profesores asociados, tanto del sector de fabricación asistida, de ingeniería mecánica y de ciencia de materiales, cubriendo así las áreas más relevantes de la ingeniería relacionadas con la impresión 3D.

\subsection{Fase segunda: Elaboración del cuestionario}

En primer lugar se realizaron entrevistas personales a profesionales del sector, buscando su punto de vista sobre el presente y futuro de los profesionales que trabajan en la fabricación aditiva. El formato de respuesta a las preguntas fue respuesta abierta. Las preguntas se agruparon en 4 áreas:

- Tendencias y desarrollos sectoriales

- ¿Consideras que la impresión 3D contribuirá a la eficiencia en la fabricación la productividad?

- ¿Piensa que tendrá un impacto en la cadena de suministro global y/o traerá cambios en los modelos de negocio existentes? En caso afirmativo, indíquelos

- ¿Qué sectores de fabricación serán más probablemente afectados por la proliferación de la impresión 3D?

- ¿En cuánto se refiere a los trabajadores de la fabricación, cuáles son, según su conocimiento, los principales puestos de trabajo y tareas que requieren habilidades de impresión 3D? ¿Cuáles son los nuevos roles emergentes?

- Habilidades y competencias

- ¿Será posible para alguien sin formación especializada hacer frente a la integración de la impresión $3 D$ en la fabricación?

- ¿Qué habilidades, competencias y conocimientos necesitan los empleados de fábrica para trabajar eficientemente con equipos de impresión 3D?

- ¿Puede listar por orden de importancia los requisitos que usted ha mencionado?

- Basado en su respuesta anterior, ¿cree que hay falta de habilidades y habilidades que no se ajustan a las necesidades?

- ¿Conoce algún plan o iniciativa de la industria para identificar y hacer frente a estos desajustes?

- Formación Profesional y Cualificaciones

- ¿En términos de cualificaciones ofrecidas, ¿los cursos existentes para los empleados de fabricación cubren las necesidades y requisitos necesarios para la integración de la impresión 3D? ¿Hay diferencias entre la formación profesional y la formación para personal en activo?

- ¿Crees que habrá cambios significativos en los cursos de FP para los trabajadores destinados a la industria? 
- En tu opinión, ¿Cómo de exhaustiva o extensa debería ser la oferta de formación profesional 3D para los empleados de fabricación? [Especifique duración, frecuencia, temas de ingeniería a cubrir, interdisciplinariedad, etc]

- ¿Cómo tendrá lugar el desarrollo de competencias para la impresión 3D? [Indique si piensa que será por iniciativa de empresas de fabricación (formación interna o bajo demanda), o sobre todo a través del desarrollo de nuevos cursos por los proveedores de cursos de FP]

- Marco de Cualificaciones Europeo (EQF) y ESCO (para quien corresponda)

- ¿Cuál es el nivel de cualificación según el marco europeo (o NQF, si procede) de los trabajadores involucrados en procesos de impresión $3 D$ ? ¿Debería ser superior al de los trabajadores involucrados en los procesos de fabricación tradicional?

- ¿Sabe si la ESCO (Clasificación Europea de Capacidades, Competencias, Cualificaciones y Ocupaciones) está planeando tener en cuando los desarrollos en fabricación aditiva respecto a las habilidades y competencias en los trabajos de fabricación?

- Se ha planeado o puesto en marcha en su país algún marco de cualificación en referencia a la fabricación aditiva, que pudiera servir de información para la ESCO?

- ¿Cree que sería útil para la formación de FP, transparencia de las cualificaciones y la movilidad el desarrollo de nuevos perfiles ocupacionales para los empleados de fabricación implicados en procesos de impresión $3 D$ ?

A partir de las respuestas de las entrevistas se generó la encuesta, cuyo propósito es la recopilación de información para desarrollar y actualizar los perfiles ocupacionales de personas que trabajan, o trabajarán, en la industria de fabricación aditiva. La encuesta se estructuró en 4 áreas, de acuerdo con las diferentes etapas del proceso del aditivo (por ejemplo la producción de fabricación, montaje y producción). Se muestran a continuación las preguntas de la encuesta, agrupadas por área. El formato de respuesta fue de respuesta múltiple, con cinco posibles respuestas (No requerido, Prioridad baja, Prioridad media, Prioridad Alta, No sabe/No contesta), donde el experto debe indicar la importancia que estima para los futuros profesionales en impresión 3D que tiene cada una de las habilidades por las que se le pregunta.

- Conocimientos requeridos para técnicos de fabricación aditiva

- Comprender los diferentes tipos de procesos de fabricación aditiva disponibles, así como sus ventajas y desventajas

- Comprender los diferentes materiales, y sus propiedades, que se utilizan para producir componentes en los procesos de fabricación aditiva

- Comprender cómo pueden ocurrir problemas y defectos en componentes producidos mediante procesos de fabricación aditiva, así como las medidas preventivas

- Comprender las técnicas de acabado (por ejemplo, mecanizado, cadena, capa, tratamiento térmico) utilizadas para las diferentes técnicas de fabricación aditiva

- Aplicación de prácticas de seguridad en el trabajo y los procedimientos en todo momento

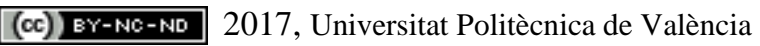


- Habilidades/competencias requeridas para la fase de preproducción.

- comprobar que todo el equipo está en condiciones de trabajo seguro

- estimación de la cantidad materiales necesarios, correcta manipulación y almacenamiento de materiales y chequeo de documentación

- obtener todos los datos necesarios, documentación y especificaciones para el componente a producir

- interpretación de dibujos, sistemas imperial y métrico, puntos de referencia de la pieza de trabajo, sistemas de tolerancias

- Chequeo de archivos de fabricación correctos para producir los componentes

- Comprobación de la adecuación de los archivos de datos

- comprensión de los principios básicos de los equipos de fabricación aditiva que se utilizan

- Habilidades/competencias requeridas para la fase de puesta en marcha.

- capacidad de iniciar o detener el equipo durante una situación de uso normal, finalización y situaciones de emergencia

- comprender los riesgos asociados con el uso de máquinas de fabricación aditiva y cómo minimizarlos

- Uso de equipos de protección apropiados y mantenimiento del área de trabajo de manera ordenada y segura

- importación de archivos correspondientes (por ejemplo STL) de un sistema de CAD a software de fabricación aditiva

- capacidad de comprobación del conjunto de parámetro de funcionamiento de equipos (acceso programa, edición, facilidad para hacer ajustes menores para la producción...)

- Habilidades/competencias para la fase de producción.

- seguimiento de parámetros del proceso para el control de calidad

- saber cuándo las piezas pueden ser retiradas de la máquina

- eliminación de material de soporte y desechos

- inspección de la pieza para el control dimensional, revisión de la apariencia, y detección de defectos visibles

- detección y definición de los componentes a limpiar, empaquetar y almacenar con toda su documentación asociada (p. ej. $N^{o}$ de lote, número de archivo CAD, fecha, operador, etc...)

- dejar la máquina en condiciones seguras y óptimas para el próximo uso, por ejemplo: cierre del programa, limpieza de la máquina, retirada y acondicionamiento de residuos

\subsection{Fase tercera: Distribución y recopilación del cuestionario}

Se realizaron entrevistas personales a candidatos relevantes. En total se llevaron a cabo 26 entrevistas en 3 países (Italia, Reino Unido y España). 
Además, una vez seleccionados los profesionales que serían encuestados, se realizó la distribución del cuestionario en línea. Se enviaron 200 cuestionarios, de los cuáles 119 resultaron contestados en su totalidad.

\subsection{Fase cuarta: Tratamiento de datos}

Como resultado de las entrevistas se pudo plantear un cuestionario adaptado a la realidad del sector.

Los resultados de las encuestas fueron tratados minuciosamente para extraer los resultados de aprendizaje que la sociedad demanda del alumno de fabricación en impresión 3D.

No se incluyen las respuestas ni de las entrevistas ni de los cuestionarios debido a su extensión, que resulta excesiva para el presente documento. Sin embargo, sí que se presentan los resultados de aprendizaje derivados del trabajo previo.

\section{Resultados}

A partir del análisis de los datos obtenidos en el cuestionario, se han identificado una serie de requisitos de conocimiento y habilidades / competencias requeridas para las fases de preproducción, puesta en marcha y producción de la implementación industrial.

En las tablas siguientes se asignan resultados de aprendizaje a cada uno de los requisitos de conocimiento, habilidades y competencias.

Tabla 1. Conocimientos previos.

\begin{tabular}{cc}
\hline Conocimientos previos & \multicolumn{1}{c}{ Resultados de aprendizaje } \\
\hline Aplicación de prácticas y & - Conocer las prácticas y procedimientos de trabajo seguros \\
procedimientos seguros de & - Comprender las consecuencias de no adoptar prácticas y \\
trabajo en todo momento & procedimientos de trabajo seguros \\
& - Saber cómo mejorar las prácticas y procedimientos de trabajo \\
& seguros si se descubre que son subóptimos \\
\hline Comprender los diferentes tipos & - Ser capaz de enumerar y describir los principios operativos de \\
de procesos de fabricación de & todos los procesos FA relevantes \\
aditivos (FA) disponibles, así & - Describir las aplicaciones de cada proceso y sus ventajas para \\
como sus ventajas y & dichos procesos \\
desventajas. & - Describir las desventajas de cada proceso para sus aplicaciones \\
\hline Comprender los diferentes & - Enumerar los materiales metálicos y no metálicos adecuados para \\
materiales, y sus propiedades,, & - Describir las propiedades físicas y químicas de cada material \\
utilizados para producir & - Estar al tanto de los requisitos de salud y seguridad para materiales \\
componentes por el proceso FA & seleccionados \\
& - Describir cualquier pretratamiento requerido para preparar cada \\
& material para FA \\
\hline
\end{tabular}

(c) EY-NC-ND 2017, Universitat Politècnica de València

Congreso IN-RED (2017) 
- FA describir cualquier post-procesamiento requerido para hacer que el producto producido por FA sea apto para el propósito

- Enumerar y describir los tipos de defectos que potencialmente

Comprender cómo los problemas y defectos pueden ocurrir en los componentes producidos por los procesos de

FA, así como las medidas preventivas. podrían surgir en cada proceso FA

- Explicar técnicas (no destructivas) para la detección de defectos

- Comprender las razones por las que se produce cada categoría de defecto

- Describir las técnicas de control de calidad, preprocesamiento, en proceso, post-proceso y otras utilizadas para prevenir o corregir problemas o defectos

- Conocer las diversas tecnologías utilizadas para el acabado (post-

Comprender las técnicas de proceso) de los productos FA

acabado (por ejemplo,

Mecanizado, HIP,

Recubrimiento, Tratamiento

Térmico) utilizadas para las diversas técnicas FA.

- Conocer los procedimientos operativos necesarios para las técnicas de acabado

- Explicar los beneficios / mejoras realizadas a los productos de FA por las operaciones de acabado

- Describir los métodos de control de calidad utilizados para comprobar la eficacia de estas técnicas de acabado

\begin{tabular}{|c|c|}
\hline $\begin{array}{l}\text { Posee habilidades de diseño } \\
\text { CAD en relación con el diseño } \\
\text { de fabricación / producto. }\end{array}$ & $\begin{array}{l}\text { - Conocer el sistema (s) CAD utilizado (s) para los productos FA } \\
\text { - Ser capaz de aplicar los sistemas CAD de manera efectiva a los } \\
\text { productos FA }\end{array}$ \\
\hline $\begin{array}{l}\text { Entienda cómo la posición y la } \\
\text { orientación afectan las } \\
\text { propiedades de la construcción. }\end{array}$ & $\begin{array}{l}\text { - Apreciar la influencia de la posición del producto en la calidad del } \\
\text { producto final de la FA } \\
\text { - Comprender los efectos de las diferentes orientaciones sobre la } \\
\text { calidad del producto } \\
\text { - Ser capaz de seleccionar la mejor combinación de posición y } \\
\text { orientación para un producto determinado }\end{array}$ \\
\hline
\end{tabular}

Tabla 2. Habilidades / competencias: fase de pre-producción.

\begin{tabular}{cc}
\hline $\begin{array}{c}\text { Habilidades / competencias: } \\
\text { fase de pre-producción }\end{array}$ & Resultados de aprendizaje \\
\hline & - Comprender las características de seguridad y procedimientos de \\
& verificación de seguridad para todos los equipos \\
Compruebar que todo el equipo & - Saber informar y/o corregir los problemas relacionados con la \\
está en condiciones de trabajo & seguridad \\
seguras y utilizables. & - Comprender los principios de funcionamiento del equipo \\
& - Saber cómo comprobar previamente todas las funciones de \\
& funcionamiento y cómo informar y/o corregir cualquier problema \\
\hline Obtener cantidades suficientes & - Conocer los materiales y cantidades requeridos para cada \\
de todos los materiales & proceso/producto \\
requeridos, manejo correcto / & - Comprender los requisitos de manipulación y almacenamiento para \\
almacenamiento de materiales y & cada material \\
revise la documentación. & - Estar familiarizado con la documentación y cómo debe ser \\
& verificada/completada \\
\hline Obtener todos los datos, & - Conocer qué datos, documentación y especificaciones se requieren \\
\hline
\end{tabular}




\begin{tabular}{|c|c|}
\hline $\begin{array}{l}\text { documentación y } \\
\text { especificaciones necesarios para } \\
\text { que el componente se produzca. }\end{array}$ & $\begin{array}{l}\text { de cada componente } \\
\text { - Conocer dónde encontrar los datos, la documentación y las } \\
\text { especificaciones pertinentes si alguno de ellos falta o está } \\
\text { incompleto }\end{array}$ \\
\hline $\begin{array}{l}\text { Descargar los archivos de } \\
\text { compilación correctos para } \\
\text { producir los componentes. }\end{array}$ & $\begin{array}{l}\text { - Conocer dónde se almacenan los archivos de compilación y cómo } \\
\text { descargarlos } \\
\text { - Comprender cómo comprobar que el archivo de compilación se } \\
\text { relaciona con el componente correcto }\end{array}$ \\
\hline $\begin{array}{l}\text { Compruebar que los archivos de } \\
\text { datos son adecuados para la } \\
\text { aplicación. }\end{array}$ & $\begin{array}{l}\text { - Estar familiarizado con las especificaciones y la nomenclatura que } \\
\text { relacionan los archivos de datos con componentes específicos } \\
\text { - Conocer cómo ejecutar en seco software para comprobar que } \\
\text { construir y archivos de datos son correctos }\end{array}$ \\
\hline $\begin{array}{l}\text { Entender los principios básicos } \\
\text { del equipo FA que se está } \\
\text { utilizando. }\end{array}$ & $\begin{array}{l}\text { - Comprender los roles físicos que desempeña cada equipo } \\
\text { - Ser capaz de describir los procesos físicos que ocurren durante el } \\
\text { ciclo FA } \\
\text { - Conocer los parámetros y sistemas de control y por qué son } \\
\text { importantes }\end{array}$ \\
\hline $\begin{array}{l}\text { La comunicación clara con los } \\
\text { compañeros de trabajo y los } \\
\text { clientes es importante para } \\
\text { asegurar que los componentes } \\
\text { se fabrican correctamente. }\end{array}$ & $\begin{array}{l}\text { - Saber quién es responsable de todas las etapas de la producción y } \\
\text { de la interacción con el cliente } \\
\text { - Elaborar una lista de verificación de la información interna y de los } \\
\text { clientes clave, sin la que no se pueda iniciar la fabricación } \\
\text { - Entender qué hacer o quién consultar si falta información clave o } \\
\text { no está claro }\end{array}$ \\
\hline $\begin{array}{l}\text { Interpretar dibujos, sistemas de } \\
\text { medida imperial y métrica, } \\
\text { puntos de referencia de piezas, } \\
\text { sistemas de tolerancia. }\end{array}$ & $\begin{array}{l}\text { - Conocer dibujo de ingeniería } \\
\text { - Ser capaz de convertir fácilmente entre sistemas de medición } \\
\text { imperial y métrica } \\
\text { - Conocer la metrología y tolerancia de ingeniería }\end{array}$ \\
\hline
\end{tabular}

Tabla 3. Habilidades / competencias: fase de puesta en marcha.

\begin{tabular}{|c|c|}
\hline $\begin{array}{l}\text { Habilidades / competencias: } \\
\text { fase de puesta en marcha }\end{array}$ & Resultados de aprendizaje \\
\hline $\begin{array}{l}\text { Capaz de arrancar / parar el } \\
\text { equipo en situaciones normales, } \\
\text { completas y de emergencia. }\end{array}$ & $\begin{array}{l}\text { - Estar familiarizado con los mecanismos y procedimientos de } \\
\text { inicio/parada de rutina } \\
\text { - Estar familiarizado con los procedimientos y consecuencias de } \\
\text { cierre de emergencia }\end{array}$ \\
\hline $\begin{array}{l}\text { Comprender los peligros, y } \\
\text { cómo se minimizan, que están } \\
\text { asociados con el } \\
\text { funcionamiento de máquinas } \\
\text { FA. }\end{array}$ & $\begin{array}{l}\text { - Comprender todos los peligros potenciales asociados con los } \\
\text { materiales utilizados en FA y cómo manejar materiales para } \\
\text { prevenir incidentes } \\
\text { - Comprender todos los peligros potenciales asociados con el } \\
\text { funcionamiento del equipo de FA y cómo prevenir incidentes a } \\
\text { través de prácticas de operación seguras }\end{array}$ \\
\hline $\begin{array}{l}\text { Use EPI's apropiado y } \\
\text { mantenga el área de trabajo } \\
\text { segura y ordenada. }\end{array}$ & $\begin{array}{l}\text { - Tener un conocimiento detallado de las EPI's } \\
\text { - Comprender los principios de "5S" (ordenar, establecer en orden, } \\
\text { brillo, estandarizar, mantener) y cómo se utilizan para establecer y } \\
\text { mantener un entorno de trabajo seguro }\end{array}$ \\
\hline Importe archivos apropiados & - Entender qué archivos son apropiados para una aplicación dada \\
\hline
\end{tabular}

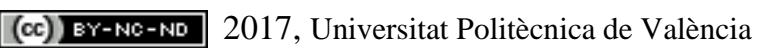




\begin{tabular}{cc}
\hline $\begin{array}{c}\text { desde un sistema de datos al } \\
\text { software FA }\end{array}$ & - Conocer importar los archivos apropiados \\
\hline Capaz de comprobar/fijar los & - Entender los parámetros operativos para una gama de producción \\
parámetros de funcionamiento & - Estar familiarizado con los procedimientos de ajuste de los \\
del equipo. & parámetros de funcionamiento \\
\hline
\end{tabular}

Tabla 4. Habilidades / competencias: fase de producción.

\begin{tabular}{|c|c|}
\hline $\begin{array}{l}\text { Habilidades / competencias: } \\
\text { fase de producción }\end{array}$ & Resultados de aprendizaje \\
\hline $\begin{array}{c}\text { Conocer cuándo se puede } \\
\text { descargar el (los) } \\
\text { componente(s) de la máquina. }\end{array}$ & $\begin{array}{l}\text { - Entender cuándo se ha completado el ciclo de producción } \\
\text { - Conocer cuándo es seguro extraer el (los) componente (s) de la } \\
\text { máquina, con respecto a la salud y seguridad personal }\end{array}$ \\
\hline $\begin{array}{l}\text { Retire la parte de la materia } \\
\text { prima restante y los soportes. El } \\
\text { almacenamiento seguro y la } \\
\text { eliminación de filtros usados y } \\
\text { material de desecho en polvo es } \\
\text { muy importante en este proceso. }\end{array}$ & $\begin{array}{l}\text { - Estar familiarizado con los procesos de extracción segura de piezas } \\
\text { y soportes } \\
\text { - Estar familiarizado con los procedimientos y requisitos de } \\
\text { seguridad para la eliminación y eliminación de filtros usados y } \\
\text { polvos de desecho } \\
\text { - Comprender los riesgos potenciales para la salud y la seguridad } \\
\text { asociados con los polvos de desecho y los filtros contaminados }\end{array}$ \\
\hline $\begin{array}{l}\text { Deje la máquina en condiciones } \\
\text { seguras al completar la } \\
\text { actividad de FA, p. Cerrar } \\
\text { programas, limpiar la máquina, } \\
\text { eliminar y eliminar residuos. }\end{array}$ & $\begin{array}{l}\text { - Estar familiarizado con todos los procedimientos para cerrar el } \\
\text { software } \\
\text { - Estar familiarizado con todos los procedimientos para apagar y } \\
\text { limpiar máquinas FA después de su uso } \\
\text { - Comprender los procedimientos para la eliminación segura de } \\
\text { materiales de desecho } \\
\text { - Comprender las consecuencias de no cumplir con los } \\
\text { procedimientos de reducción prescritos }\end{array}$ \\
\hline $\begin{array}{l}\text { Consciente de qué soporte está } \\
\text { disponible de los ingenieros de } \\
\text { servicio y los ingenieros de } \\
\text { aplicaciones. }\end{array}$ & $\begin{array}{l}\text { - Conocer los parámetros de control del proceso y la forma en que se } \\
\text { controlan / miden } \\
\text { - Conocer, registrar y reportar parámetros de control de procesos } \\
\text { - Reconocer cuando los parámetros de control del proceso están } \\
\text { fuera de especificación y qué hacer al respecto }\end{array}$ \\
\hline $\begin{array}{l}\text { Supervisión de los parámetros } \\
\text { del proceso para el control de } \\
\text { calidad. }\end{array}$ & $\begin{array}{l}\text { - Estar familiarizado con las especificaciones / normas para las } \\
\text { dimensiones de los componentes, defectos y apariencia } \\
\text { - Comprender el equipo y procedimientos utilizados para el control } \\
\text { de calidad de los componentes }\end{array}$ \\
\hline $\begin{array}{l}\text { nspección del componente para } \\
\text { precisión dimensional, defectos } \\
\text { visibles y apariencia. }\end{array}$ & $\begin{array}{l}\text { - Entender perfectamente los equipos y procedimientos para limpiar } \\
\text { los componentes, empaquetarlos y almacenarlos } \\
\text { - Estar familiarizado con la documentación de los registros de } \\
\text { componentes y clientes }\end{array}$ \\
\hline $\begin{array}{l}\text { Componentes a limpiar, } \\
\text { empaquetar y almacenar con } \\
\text { toda la documentación } \\
\text { necesaria (por ejemplo, número } \\
\text { de lote, número de archivo...) }\end{array}$ & $\begin{array}{l}\text { - Estar familiarizado con los equipos y procedimientos utilizados } \\
\text { para el mantenimiento de máquinas FA } \\
\text { - Conocer cómo operar el equipo de acuerdo con los procedimientos } \\
\text { prescritos }\end{array}$ \\
\hline
\end{tabular}




\section{Conclusiones}

La definición de los objetivos de aprendizaje de un programa formativo es de gran interés, pues permite generar la estructura de un curso que pueda adaptarse tanto a un sistema basado en ECTS como a uno basado en ECVET.

Con el apoyo de las políticas de la UE para la cualificación, la transparencia, la movilidad y el renacer de la industria europea, se han identificado las competencias que el alumnado ha de cubrir durante su formación, en cada uno de los estadios de la misma.

En este trabajo se han establecido los resultados de aprendizaje en la formación sobre impresión 3D, a partir del dialogo Universidad-Sociedad. En primer lugar se realizaron entrevistas personales a profesionales de varios países de la Unión Europea (Italia, Reino Unido y España). El análisis de las respuestas (en formato respuesta abierta) ha conducido a la elaboración de un cuestionario con preguntas (en formato respuesta múltiple) que permiten determinar la percepción de la importancia de las distintas competencias por los profesionales del sector de la impresión 3D. En total se han obtenido un total de 119 encuestas completas, siendo esta información una base robusta para la enumeración de los objetivos de aprendizaje del programa formativo.

Este trabajo, por lo tanto, ha permitido establecer unos objetivos de aprendizaje perfectamente adaptados a la realidad profesional. En el desarrollo del proyecto Europeo 3D-PRISM, estos objetivos de aprendizaje servirán para el desarrollo de un curso en impresión 3D.

\section{Agradecimientos}

Los autores desean agradecer el apoyo financiero recibido a través del proyecto ERASMUS +: 3D PRINTING SKILLS FOR MANUFACTURIN G 2015-1-UK01-KA202-013432.

\section{Referencias}

CEDEFOP-EUROPEAN CENTRE FOR THE DEVELOPMENT OF VOCATIONAL TRAINING (2016a) Application of learning outcomes approaches across Europe. A comparative study. Cedefop reference series No $105 . \quad<\mathrm{http} / / / \mathrm{www} . c e d e f o p . e u r o p a . e u / e n / p u b l i c a t i o n s-a n d-$ resources/publications/3074> [Consulta: 20 de mayo de 2017]

CEDEFOP-EUROPEAN CENTRE FOR THE DEVELOPMENT OF VOCATIONAL TRAINING (2016b) ECVET in Europe: monitoring report 2015. Cedefop research paper No 56 <http://www.cedefop.europa.eu/en/publications-and-resources/publications/5556> [Consulta: 20 de mayo de 2017]

CEDEFOP-EUROPEAN CENTRE FOR THE DEVELOPMENT OF VOCATIONAL TRAINING (2013) Monitoring ECVET implementation strategies in Europe. Cedefop working Paper No 18. <www.cedefop.europa.eu/files/6118_en.pdf> [Consulta: 20 de mayo de 2017]

(cc) EY-NC-ND 2017, Universitat Politècnica de València 
EUROPEAN COMMISSION-EDUCATION AND CULTURE DG (2011) Using learning outcomes European Qualification Framework series: Note 4. <http://www.cedefop.europa.eu/en/news-andpress/news/using-learning-outcomes $>$ [Consulta: 20 de mayo de 2017]

EUROPEAN COMMISSION-EDUCATION AND TRAINING (2014) Implementation of the Recommendation of the European Parliament and of the Council of 18 June 2009 on the establishment of a European Credit System for Vocational Education and Training (ECVET). <http://ec.europa.eu/dgs/education_culture/more_info/evaluations/docs/education/ecvet14_en.pdf > [Consulta: 20 de mayo de 2017]

EUROPEAN COMMUNITIES (2008). The European Qualifications Framework <http://eurlex.europa.eu/legal-content/EN/TXT/PDF/?uri=CELEX:32008H0506(01)\&from=EN> [Consulta: 20 de mayo de 2017]

EUROPEAN PARLIAMENT; COUNCIL OF THE EUROPEAN UNION (2009) Recommendation of the European Parliament and the Council of 18 June 2009 on the establishment of a European credit system for vocational education and training (ECVET). Official Journal of the European Union. <http://eur-lex.europa.eu/legal-content/EN/ALL/?uri=CELEX\%3A32009H0708(02> [Consulta: 20 de mayo de 2017]

GONCALVES, M., PIMENTA, P., BRAGA, L. y COTA, M. (2013) "Defining and Classifying Learning Outcomes: A Case Study" en Proceedings of the Informing Science and Information Technology Education Conference, vol. 2013, issues 1, p. 209-226. 